ШSOLÍDS

\title{
Solutions to the Eshelby Conjectures
}

Liping Liu

\author{
November 28, 2007
}




\title{
Solutions to the Eshelby conjectures
}

\author{
By L.P. LIU
}

${ }^{1}$ Division of Engineering and Applied Science, California Institute of Technology, Pasadena, CA 91125, USA

\begin{abstract}
We present solutions to the Eshelby conjectures based on a variational inequality. We first discuss the meanings of the original Eshelby's statement. By Fourier analysis, we establish the connection between the homogeneous Eshelby inclusion problem and the classic Newtonian potential problem. We then proceed to the solutions of the Eshelby conjectures. Under some hypothesis on the material properties and restricted to connected inclusions with Lipschitz boundary, we show that one version of the Eshelby conjectures is valid in all dimensions and the other version is valid in two dimensions. We also show the existence of multiply-connected inclusions in all dimensions and the existence of non-ellipsoidal connected inclusions in three and higher dimensions such that, in physical terms and in the context of elasticity, some uniform eigenstress of the inclusion induces uninform strain on the inclusion. We numerically calculate these special inclusions based on the finite element method.
\end{abstract}

\section{Introduction}

The following remarkable property of ellipsoids was first observed by Poisson (1826): given a uniformly magnetized/polarized ellipsoid, the induced magnetic/electric field is also uniform inside the ellipsoid. Explicit expressions for this field were obtained by Maxwell (1873). A similar result also occurs in linearized elasticity, where the Eshelby's solution asserts that a uniform eigenstress on an ellipsoidal inclusion in an infinite elastic medium induces uniform strain inside the ellipsoid (Eshelby 1957, 1961; Mura 1987). In a general setting this remarkable property of ellipsoids can be summarized as the following theorem.

Theorem 1.1. Let $\mathbf{L}: \mathbb{R}^{m \times n} \rightarrow \mathbb{R}^{m \times n}$ be either self-adjoint and positive definite or an elasticity tensor with the usual symmetries, $\Omega \subset \mathbb{R}^{n}$ be an inclusion, and $\chi_{\Omega}$ be the characteristic function of $\Omega$. Let $\mathbf{v} \in W_{\text {loc }}^{1,2}\left(\mathbb{R}^{n}, \mathbb{R}^{m}\right)$ be a solution of

$$
\operatorname{div}\left[\mathbf{L} \nabla \mathbf{v}+\mathbf{P} \chi_{\Omega}\right]=0 \quad \text { on } \mathbb{R}^{n}
$$

in the sense that

$$
[\nabla \mathbf{v}(\mathbf{x})]_{p i}=\frac{-1}{(2 \pi)^{n}} \int_{\mathbb{R}^{n}} N_{p q}(\mathbf{k})(\mathbf{P})_{q j}(\mathbf{k})_{j}(\mathbf{k})_{i} \int_{\Omega} \exp \left(i \mathbf{k} \cdot\left(\mathbf{x}-\mathbf{x}^{\prime}\right)\right) d \mathbf{x}^{\prime} d \mathbf{k}
$$

where $N_{p q}(\mathbf{k})$ is the inverse of the matrix $(\mathbf{L})_{p i q j}(\mathbf{k})_{i}(\mathbf{k})_{j}$. If $n=2,3$ and $\Omega$ is an ellipsoidal inclusion, then $\nabla \mathbf{v}$ is uniform on $\Omega$ for any $\mathbf{P} \in \mathbb{R}^{m \times n}$. 
In equation (1.2) and subsequently, the Einstein summation convention is followed. Throughout this paper, we mean by the term "inclusion" an open and bounded domain which may have several separated components. Obviously, the representation formula in (1.2) follows from Fourier analysis, see Khachaturyan (1983) and Mura (1987). Below, we sometimes write a solution of (1.1) as $\mathbf{v}(\mathbf{x}, \mathbf{P})$ to emphasize the (linear) dependence of $\mathbf{v}$ on $\mathbf{P}$. A proof of Theorem 1.1 can be adapted from the calculations in Mura (1987, Ch. 3), see also Asaro and Barnett (1975). Note that equation (1.1) covers the physical problems mentioned above. In electrostatics/magnetostatics, $m=1$ and equation (1.1) determines the electric/magnetic field $\nabla \mathbf{v}$ induced by a uniform polarization/magnetization $\mathbf{P}$ on $\Omega$ with permitivity/permeability tensor L. In linearized elasticity equation (1.1) is referred to as the homogeneous Eshelby inclusion problem, where $\mathbf{L}, \mathbf{v}$ and $\mathbf{P}$ represent elasticity tensor, displacement and eigenstress, respectively. Since $\nabla \mathbf{v}$ being constant on $\Omega$ leads to great simplification, ellipsoidal inclusions play a central role in the theory of composites (Christensen 1979; Milton 2002), in micromechanics (Mura 1987) and in experimental measurements (Brown 1962). The uniformity of the induced field can also be used to solve the minimization problems that arise in the theories of ferroelectric and magnetostrictive materials, see Desimone and James (2002), Bhattacharya and Li (2001), and Liu et al (2006). To extend these analysis, a natural question arises: are there any other inclusions having this uniformity property? Eshelby (1961) conjectured:"......Among closed surfaces, the ellipsoid alone has this convenient property......". One can take this statement to mean the following:

(i) For an inclusion $\Omega \subset \mathbb{R}^{3}$, if the induced field $\nabla \mathbf{v}(\mathbf{x}, \mathbf{P})$ defined by (1.2) is uniform on $\Omega$ for a single nonzero $\mathbf{P} \in \mathbb{R}^{3 \times 3}$, then $\Omega$ must be an ellipsoid.

(ii) For an inclusion $\Omega \subset \mathbb{R}^{3}$, if the induced field $\nabla \mathbf{v}(\mathbf{x}, \mathbf{P})$ defined by (1.2) is uniform on $\Omega$ for any $\mathbf{P} \in \mathbb{R}^{3 \times 3}$, then $\Omega$ must be an ellipsoid.

In the context of Eshelby (1961), the tensor $\mathbf{L}$ in (1.1) is an isotropic elasticity tensor. Naturally we generalize these conjectures to other positive semi-definite tensors and other dimensions. For future convenience, we refer to statement (i) and (ii) as the Eshelby conjecture I and the Eshelby conjecture II, respectively. Clearly the Eshelby conjecture I implies the Eshelby conjecture II. It appears that many authors tacitly choose the second meaning of the Eshelby's statement and quote it as the Eshelby conjecture, see e.g. Mura (2000) and Markenscoff (1998a). Various authors have tried to prove or disprove the Eshelby conjecture. For instance, Mura et al (1994) (see also Mura 2000) claimed that certain pentagonal star-shaped domains share this remarkable property with ellipsoids, which was later pointed out by Rodin (1996) and Markenscoff (1998a) false. Markenscoff (1998a) showed that the domains in $\mathbb{R}^{3}$ with this uniformity property, considered in a proper space, have to be closed and form a 9-dimensional manifold. She also showed that any shape with a planar piece on its boundary cannot have this property (Markenscoff 1998b). Meanwhile, all other known solutions for non-ellipsoidal inclusions do not contradict the Eshelby conjectures, see Lee and Johnson (1977) for solutions of cuboidal inclusions, Wu and Du (1995) for solutions of circular cylinders, and Rodin (1996) for solutions of polyhedra. The Eshelby conjectures were proved by complex variables method in two dimensions, see Sendeckyj (1970) and Ru and Schiavone (1996). All these evidences suggest that the Eshelby conjectures, especially the 
second version, would likely be true in any dimension. The main difficulty of a proof arises from the nonlocal dependence of $\nabla \mathbf{v}$ on $\Omega$, which is governed by the partial differential equation (1.1). Therefore, it is hard to verify if $\nabla \mathbf{v}$ is exactly uniform on $\Omega$ for a given inclusion $\Omega$.

In this paper we present solutions to the Eshelby conjecture interpreted in either sense. We overcome the aforementioned difficulty by considering a variational inequality. Roughly speaking, instead of calculating the induced field $\nabla \mathbf{v}$ for a given inclusion $\Omega$, we prescribe the field $\nabla \mathbf{v}$ and then construct the inclusion $\Omega$ such that it gives rise to this field. In this way we are able to show the validity of the Eshelby conjecture II if restricted to connected inclusions with Lipschitz boundaries. Moreover, we can construct simply-connected non-ellipsoidal inclusions in three dimensions and multiply-connected inclusions in all dimensions having uniform fields $\nabla \mathbf{v}$ on the inclusions for various matrices $\mathbf{P}$. The existence of such simply-connected non-ellipsoidal inclusions shows that the validity of the Eshelby conjecture I in general depends on the tensor $\mathbf{L}$ and the matrix $\mathbf{P}$ in three or higher dimensions even if restricted to connected inclusions with Lipschitz boundaries.

To proceed, we shall require that

$$
m=n \quad \text { and } \quad(\mathbf{L})_{p i q j}=\mu_{1} \delta_{i j} \delta_{p q}+\mu_{2} \delta_{p j} \delta_{i q}+\lambda \delta_{i p} \delta_{j q},
$$

where $\delta_{i j}(i, j=1, \cdots, n)$ are the components of the identity matrix $\mathbf{I}$. The constants $\mu_{1}, \mu_{2}, \lambda$ are always required to satisfy

$$
\mu_{1} \geq \mu_{2}, \quad \mu_{1}+\mu_{2}>0 \quad \text { and } \quad \lambda>-\left(\mu_{1}+\mu_{2}\right) / n,
$$

which ensures $\mathbf{L}$ is either positive definite or an isotropic elasticity tensor. It is worthwhile noticing that tensors of this form cover the most common situations in the physical problems discussed above. In particular,

(i) $\mu_{1}=\mu_{2}=\mu>0$ corresponds to isotropic elasticity tensors, and

(ii) $\mu_{2}=\lambda=0$ corresponds to isotropic permittivity/permeability tensors in electrostatic/magnetostatic problems. In fact, each component in the vector $\mathbf{u}$ is the potential induced by the polarization/ magnetization of the corresponding row vector in the matrix $\mathbf{P}$.

We now state the main results of this paper.

Theorem 1.2. If $n \geq 2, \mathbf{L}$ is given by (1.3) and (1.4), and if restricted to connected inclusions with Lipschitz boundaries, the Eshelby conjecture II holds. More precisely, if an inclusion $\Omega$ is connected and $\partial \Omega$ is Lipschitz continuous, and if equation (1.1) with $\mathbf{L}$ specified by (1.3) and (1.4) has a solution $\mathbf{v}(\mathbf{x}, \mathbf{P})$ satisfying

$$
\nabla \mathbf{v}(\mathbf{x}, \mathbf{P})=\text { const. } \quad \text { on } \Omega \quad \forall \mathbf{P} \in \mathbb{R}^{n \times n},
$$

then $\Omega$ must be an ellipsoidal inclusion.

Theorem 1.3. If $n=2, \mathbf{L}$ is given by (1.3) and (1.4), and if restricted to connected inclusions with Lipschitz boundaries, the Eshelby conjecture I holds. More precisely, if an inclusion $\Omega$ is connected and $\partial \Omega$ is Lipschitz continuous, and if equation (1.1) with $\mathbf{L}$ specified by (1.3) and (1.4) has a solution $\mathbf{v}(\mathbf{x}, \mathbf{P})$ satisfying

$$
\nabla \mathbf{v}(\mathbf{x}, \mathbf{P})=\text { const. } \quad \text { on } \Omega \text { for a single nonzero } \mathbf{P} \in \mathbb{R}^{n \times n},
$$

then $\Omega$ must be an ellipsoidal inclusion. 
Theorem 1.3 has been proved by Sendeckyj (1970), see also Ru and Schiavone (1996). We recently learned of works of Kang and Milton (2007) who proved Theorem 1.2 for $n=3$, see also Dive (1931) and Nikliborc (1932). They also observed that the Pólya-Szegö conjecture (Pólya \& Szegö 1951) is equivalent to the Eshelby conjecture II for $\mathbf{L}$ specified as in (1.3). Also, based on previous work of Cherepanov (1974), they found a class of two-component two-dimensional inclusions with the special property described in Theorem 1.4, see Kang et al (2007). We remark that our work is simultaneous and independent from theirs. In particular, we are able to construct the following examples, which show that the requirement of $\Omega$ being connected in Theorem 1.2 and the condition $n=2$ in Theorem 1.3 are indispensable.

Theorem 1.4. Consider equation (1.1) with $\mathbf{L}$ specified by (1.3) and (1.4). There exist multiply-connected inclusions $\Omega \subset \mathbb{R}^{n}(n \geq 2)$ such that

(i) The induced field $\nabla \mathbf{v}(\mathbf{x}, \mathbf{P})$ is uniform on $\Omega$ for any $\mathbf{P} \in \mathbb{R}^{n \times n}$ if $\mu_{2}+\lambda=0$;

(ii) The induced field $\nabla \mathbf{v}(\mathbf{x}, \mathbf{I})$ is uniform on $\Omega$ for the identity matrix $\mathbf{I} \in \mathbb{R}^{n \times n}$ if $\mu_{2}+\lambda \neq 0$.

Theorem 1.5. If $n \geq 3$, the Eshelby conjecture I may or may not be valid, depending on the tensor $\mathbf{L}$ and the matrix $\mathbf{P} \in \mathbb{R}^{n \times n}$. More specifically, if an inclusion $\Omega$ is connected and $\partial \Omega$ is Lipschitz continuous, and if equation (1.1) with $\mathbf{L}$ specified by (1.3) and (1.4) with $\mu_{2}+\lambda=0$ (i.e., $\mathbf{L}: \mathbb{R}^{n \times n} \rightarrow \mathbb{R}^{n \times n}$ is the identity mapping) has a solution $\mathbf{v}(\mathbf{x}, \mathbf{P})$ satisfying

$$
\nabla \mathbf{v}(\mathbf{x}, \mathbf{P})=\text { const. } \quad \text { on } \Omega \text { for a single nonzero } \mathbf{P} \in \mathbb{R}^{n \times n},
$$

(i) if $\mathbf{P}=\mathbf{I}, \Omega$ must be an ellipsoidal inclusion;

(ii) if $\mathbf{P}=\operatorname{diag}(1,0, \cdots, 0), \Omega$ may not be an ellipsoidal inclusion, see the counterexample in Section 3(d).

The paper is organized as follows. In Section 2 we introduce a variational inequality and explain how it is related with the Eshelby conjectures. From the established theory of variational inequalities, we obtain the key existence and uniqueness Theorem 2.3. Based on Theorem 2.3, we prove the Eshelby conjecture I and II (Theorem 1.2 and Theorem 1.3) in Sections 3(a) and 3(b), respectively. We prove Theorem 1.4 and Theorem 1.5 in Section 3(c) and Section 3(d), respectively. A numerical scheme is described in Section 3(c) and is used to calculate various special inclusions in Section 3(c) and Section 3(d). A similar scheme has been verified and applied to calculate periodic E-inclusions in Liu et al (2007). Finally in Section 4 we summarize our results and propose a few applications.

\section{A related variational inequality}

In this section, we first explain the relation between equation (1.1) and the classic Newtonian potential problem. Let $\Gamma(\mathbf{x})=\Gamma(|\mathbf{x}|)$ be the fundamental solution of the Laplace operator on $\mathbb{R}^{n}$

$$
\Gamma(\mathbf{x})=\left\{\begin{array}{ll}
\frac{1}{2 \pi} \log (|\mathbf{x}|) & \text { if } n=2 \\
\frac{1}{n(2-n) \omega_{n}} \frac{1}{|\mathbf{x}|^{n-2}} & \text { if } n \geq 3
\end{array},\right.
$$

Article submitted to Royal Society 
where $\omega_{n}$ denotes the volume of a unit ball in $\mathbb{R}^{n}$. Following (4.2) of Gilbarg and Trudinger (1983), we call $u(\mathbf{x})=-\int_{\mathbb{R}^{n}} \Gamma(\mathbf{x}-\mathbf{y}) \chi_{\Omega}(\mathbf{y}) d \mathbf{y}$ the Newtonian potential induced by the source $-\chi_{\Omega}$. Clearly the Newtonian potential satisfies the Poisson equation

$$
\Delta u(\mathbf{x})=-\chi_{\Omega}(\mathbf{x}) \quad \text { on } \mathbb{R}^{n} .
$$

By Fourier analysis, the second gradient of the Newtonian potential $u(\mathbf{x})$ can be represented as

$$
[\nabla \nabla u(\mathbf{x})]_{i j}=\frac{-1}{(2 \pi)^{n}} \int_{\mathbb{R}^{n}} \frac{(\mathbf{k})_{j}(\mathbf{k})_{i}}{|\mathbf{k}|^{2}} \int_{\mathbb{R}^{n}} \chi_{\Omega}\left(\mathbf{x}^{\prime}\right) \exp \left(i \mathbf{k} \cdot\left(\mathbf{x}-\mathbf{x}^{\prime}\right)\right) d \mathbf{x}^{\prime} d \mathbf{k} .
$$

Due to the special form of $\mathbf{L}$ (cf., (1.3)), $\nabla \nabla u$ in (2.3) are closely related with $\nabla \mathbf{v}$ in (1.2). To see this, we calculate the inverse of $(\mathbf{L})_{p i q j}(\mathbf{k})_{i}(\mathbf{k})_{j}$ as

$$
N_{p q}(\mathbf{k})=\frac{1}{\mu_{1}|\mathbf{k}|^{2}} \delta_{p q}-\frac{\mu_{2}+\lambda}{\mu_{1}\left(\lambda+\mu_{1}+\mu_{2}\right)} \frac{(\mathbf{k})_{p}(\mathbf{k})_{q}}{|\mathbf{k}|^{4}}
$$

whence $(\mathbf{L})_{p i q j}(\mathbf{k})_{i}(\mathbf{k})_{j}=\mu_{1}|\mathbf{k}|^{2} \delta_{p q}+\left(\mu_{2}+\lambda\right)(\mathbf{k})_{p}(\mathbf{k})_{q}$. Comparing equation (1.2) with (2.3), we immediately have

Lemma 2.1. Consider equation (1.1) with $\mathbf{L}$ specified by (1.3) and (1.4) and the Newtonian potential problem (2.2). Let $\nabla \mathbf{v}(\mathbf{x}, \mathbf{P})$ and $\nabla \nabla u(\mathbf{x})$ be given by (1.2) and (2.3), respectively.

(i) For any inclusion $\Omega$ and $\mathbf{P}=\mathbf{I}$, we have

$$
\nabla \mathbf{v}(\mathbf{x}, \mathbf{I})=\nabla \nabla u(\mathbf{x}) /\left(\mu_{1}+\mu_{2}+\lambda\right) .
$$

(ii) If in particular $\mu_{2}+\lambda=0$, then for any $\mathbf{P} \in \mathbb{R}^{n \times n}$, we have

$$
\nabla \mathbf{v}(\mathbf{x}, \mathbf{P})=\mathbf{P} \nabla \nabla u(\mathbf{x}) / \mu_{1} .
$$

From Lemma 2.1, we see that the uniformity of $\nabla \mathbf{v}(\mathbf{x}, \mathbf{I})$ on $\Omega$ is equivalent to the uniformity of $\nabla \nabla u$ on $\Omega$. Of course, for the Newtonian potential problem (2.2), $\nabla \nabla u$ being uniform on $\Omega$ is an overdetermined condition and cannot be true unless $\Omega$ is very special. From Theorem 1.1, we know that ellipsoids enjoy such property.

To construct special inclusions such that certain overdetermined problem admits a solution, we consider the following variational inequality

$$
G_{r}\left(u_{r}\right)=\inf _{v \in K_{r}}\left\{G_{r}(v) \equiv \int_{B_{r}} \frac{1}{2}|\nabla v|^{2} d \mathbf{x}\right\},
$$

where $B_{r} \subset \mathbb{R}^{n}(n \geq 2)$ is the open ball centered at the origin of radius $r$, and for a given function $\phi: \mathbb{R}^{n} \rightarrow \mathbb{R}$ called the obstacle, the admissible set

$$
K_{r}:=\left\{v-g_{n}(r) \in W_{0}^{1,2}\left(B_{r}\right): v \geq \phi \text { on } B_{r}\right\} .
$$

Here $g_{n}:(0, \infty) \rightarrow \mathbb{R}$ is defined as $(Q>0$ is fixed)

$$
g_{n}(r)=\left\{\begin{array}{ll}
-Q \log r, & \text { if } n=2 \\
0 & \text { if } n \geq 3
\end{array} .\right.
$$

Note that $g_{n}(r)$ is a constant for fixed $r$. In the following discussions, we restrict ourselves to obstacles $\phi: \mathbb{R}^{n} \rightarrow \mathbb{R}$ with the following properties: 
(i) $\phi \in C^{0,1}\left(\mathbb{R}^{n}\right)$, there exists $R_{0}>0$ such that for some $0<R_{0}^{\prime}<R_{0}, \phi(\mathbf{x})<$ $g_{n}(|\mathbf{x}|)-\alpha\left(R_{0}^{\prime}\right)$ for all $|\mathbf{x}| \geq R_{0}$, where

$$
\alpha\left(R_{0}^{\prime}\right)=\left\{\begin{array}{ll}
\max \left\{|\phi(\mathbf{x})|:|\mathbf{x}|=R_{0}^{\prime}\right\}+\left|g_{n}\left(R_{0}^{\prime}\right)\right| & \text { if } n=2 \\
0 & \text { if } n \geq 3
\end{array} ;\right.
$$

(ii) $|\Delta \phi|$ is essentially bounded on $B_{R_{0}} \backslash U^{*}$, where $U^{*}$ is the set of singular points on which $|\nabla \nabla \phi|$ is unbounded in distributional sense, and

(iii) For all unit vector $\xi \in \mathbb{R}^{n}, \partial^{2} \phi / \partial \xi^{2}>-C$ on $\mathbb{R}^{n}$ in the sense of distributions, where $\partial / \partial \xi$ denotes the directional derivative. In another word,

$$
\int \frac{\partial^{2} \varphi}{\partial \xi^{2}}\left(\phi+\frac{1}{2} C|\mathbf{x}|^{2}\right) d \mathbf{x} \geq 0
$$

for any $\varphi \in C_{c}^{\infty}\left(\mathbb{R}^{n}\right)=\{$ smooth functions with compact support $\}$, see Friedman (1982, page 27).

We use the variational inequality (2.7) to find the minimizer $u_{r}$ and the coincident set $\left\{\mathbf{x} \in B_{r}: u_{r}(\mathbf{x})=\phi(\mathbf{x})\right\}$, and then we pass to the limit $r \rightarrow \infty$ to establish the existence of special inclusions such that certain overdetermined problem admits a solution. Similar arguments of this type can be found in Liu et al (2007). For the convenience of the reader, we present the details of the arguments below which treat general obstacles and include the case $n=2$.

First, let us recall from the established theory (Kinderlehrer and Stampacchia 1980, page 129; Friedman 1982, page 31) the following existence and regularity theorem.

Theorem 2.2. For the obstacle $\phi$ specified above, the variational inequality (2.7) has a unique minimizer $u_{r} \in W^{2, \infty}\left(B_{r}\right) \cap K_{r}$ for each $r \geq R_{0}$. Further, the unique minimizer satisfies

(i) $\phi \leq u_{r} \leq \sup \left\{\phi(\mathbf{x}): \mathbf{x} \in B_{r}\right\}$ on $B_{r}$;

(ii) The boundary of the coincident set $\Omega_{r}:=\left\{\mathbf{x} \in B_{r}: u_{r}(\mathbf{x})=\phi(\mathbf{x})\right\}$ has measure zero in $\mathbb{R}^{n}$, and

(iii) There exists a constant $C>0$, independent of $r$, such that

$$
\left\|\nabla \nabla u_{r}\right\|_{L^{\infty}\left(B_{r}\right)}<C .
$$

By choosing appropriate test functions (Friedman 1982, page 6), it can be shown that the minimizer $u_{r}$ satisfies

$$
-\Delta u_{r} \geq 0, \quad u_{r} \geq \phi, \quad \text { and } \quad-\Delta u_{r}\left(u_{r}-\phi\right)=0 \quad \text { a.e. on } B_{r} \text {. }
$$

Thus, the minimizer $u_{r}$ in fact solves the following overdetermined problem

$$
\begin{cases}\Delta u_{r}=\chi_{\Omega_{r}} \Delta \phi & \text { a.e. on } B_{r} \\ \nabla \nabla u_{r}=\nabla \nabla \phi & \text { on } \Omega_{r} \backslash \partial \Omega_{r} \\ u_{r}=g_{n}(r) & \text { on } \partial B_{r}\end{cases}
$$

Article submitted to Royal Society 
A limiting minimizer of problem (2.7) can be defined as follows. Let $r_{j} \rightarrow+\infty$ be an increasing sequence. From the properties (i) and (iii) of $u_{r}$ in Theorem 2.2, it follows that for any $r>R>R_{0}$, there is a constant $M$, independent of $r$, such that

$$
\left\|u_{r}\right\|_{W^{2, \infty}\left(B_{R}\right)} \leq M .
$$

Since $u_{r_{j}}$ is uniformly bounded in $W^{2, \infty}\left(B_{R}\right)$ for fixed $R>R_{0}$, there exists $u_{\infty} \in$ $W^{2, \infty}\left(B_{R}\right)$ such that, up to a subsequence and without relabeling,

$$
u_{r_{j}} \rightarrow u_{\infty} \text { weakly }^{*} \text { in } W^{2, \infty}\left(B_{R}\right) .
$$

From (2.11) and (2.14), we can verify that

$$
-\Delta u_{\infty} \geq 0, u_{\infty} \geq \phi, \quad \text { and } \quad-\Delta u_{\infty}\left(u_{\infty}-\phi\right)=0 \text { a.e. on } B_{R} \text {. }
$$

In particular, the first two of (2.15) follow from linearity, while the third of (2.15) is justified by the uniform convergence of $u_{r_{j}} \rightarrow u_{\infty}$. In fact, we can repeat this argument for a sequence of larger and larger values of $R$, each time taking further subsequences of $u_{r_{j}}$, and thereby obtain a function $u_{\infty} \in W_{l o c}^{2, \infty}\left(\mathbb{R}^{n}\right)$ satisfying (2.14) and (2.15) for any $R>R_{0}$. Note that equation (2.15) implies that the coincident set $\Omega_{\infty}:=\left\{\mathbf{x} \in \mathbb{R}^{n}: u_{\infty}(\mathbf{x})=\phi(\mathbf{x})\right\} \subset B_{R_{0}}$ has the property that $\left|\partial \Omega_{\infty}\right|=0$, see Friedman (1982, page 154).

We claim that $u_{\infty}$ solves the following overdetermined problem:

$$
\left\{\begin{array}{lr}
\Delta u_{\infty}=\chi_{\Omega_{\infty}} \Delta \phi & \text { a.e. on } \mathbb{R}^{n} \\
\nabla \nabla u_{\infty}=\nabla \nabla \phi & \text { on } \Omega_{\infty} \backslash \partial \Omega_{\infty} \\
\left|u_{\infty}(\mathbf{x})-g_{n}(|\mathbf{x}|)\right| \leq \frac{C_{0}}{|\mathbf{x}|^{-2}} & \text { for } \quad|\mathbf{x}| \geq R_{0}
\end{array}\right.
$$

for some $C_{0}>0$ that is independent of $\mathbf{x}$. The first two equations in (2.16) are consequences of the last equation in (2.15) and the definition of the coincident set $\Omega_{\infty}$ with $\left|\partial \Omega_{\infty}\right|=0$.

To justify the last equation in (2.16), we notice that, by the maximum principle applied to the first of (2.11), the minimum of $u_{r}(\mathbf{x})$ must be attained at $\partial B_{r}$ which implies $u_{r}(\mathbf{x}) \geq g_{n}(r)$ on $B_{r}$. From equation (2.9) and property (i) of the obstacle, it immediately follows that if $n \geq 3$, the coincident set $\Omega_{r}$ is contained in the open ball $B_{R_{0}}$ for all $r>R_{0}$. We now show this is also true for $n=2$. If $n=2$, we note that $\Delta\left[u_{r}(\mathbf{x})-g_{n}(|\mathbf{x}|)\right]=\Delta u_{r}(\mathbf{x}) \leq 0$ on $B_{r} \backslash B_{R_{0}^{\prime}}$ for any $0<R_{0}^{\prime}<R_{0}$. Also, $u_{r}(\mathbf{x})-g_{n}(|\mathbf{x}|)=0$ on $\partial B_{r}$ and $\left|u_{r}(\mathbf{x})-g_{n}(|\mathbf{x}|)\right| \leq \sup \{|\phi(\mathbf{x})|$ : $\left.\mathbf{x} \in \partial B_{R_{0}^{\prime}}\right\}+\left|g_{n}\left(R_{0}^{\prime}\right)\right|=\alpha\left(R_{0}^{\prime}\right)$ on $\partial B_{R_{0}^{\prime}}$. From the maximum principle applied to $u_{r}(\mathbf{x})-g_{n}(|\mathbf{x}|)$ restricted to $B_{r} \backslash B_{R_{0}^{\prime}}$, we conclude that $u_{r}(\mathbf{x}) \geq g_{n}(|\mathbf{x}|)-\alpha\left(R_{0}^{\prime}\right)$ on $B_{r} \backslash B_{R_{0}^{\prime}}$. By property (i) of the obstacle, we have that the coincident set $\Omega_{r} \subset B_{R_{0}}$ for $n=2$ and all $r>R_{0}$.

Further, we recall the Dirichlet Green's function for $B_{r}$ (Gilbarg and Trudinger 1983, page 19)

$G_{r}(\mathbf{x}, \mathbf{y})=\left\{\begin{array}{ll}\frac{1}{2 \pi}\left[\log (|\mathbf{y}-\mathbf{x}|)-\log \left(\sqrt{\left(\frac{|\mathbf{x}||\mathbf{y}|}{r}\right)^{2}+r^{2}-2 \mathbf{x} \cdot \mathbf{y}}\right)\right] & \text { if } n=2 \\ \frac{1}{n(2-n) \omega_{n}}\left[\frac{1}{|\mathbf{y}-\mathbf{x}|^{n-2}}-\left(\sqrt{\left(\frac{|\mathbf{x}||\mathbf{y}|}{r}\right)^{2}+r^{2}-2 \mathbf{x} \cdot \mathbf{y}}\right)^{2-n}\right] & \text { if } n \geq 3\end{array}\right.$. 
From the first and third equations in (2.12) we can express $u_{r}$ as

$$
u_{r}(\mathbf{x})=g_{n}(r)+\int_{\mathbb{R}^{n}} G_{r}(\mathbf{x}, \mathbf{y}) \Delta \phi(\mathbf{y}) \chi_{\Omega_{r}}(\mathbf{y}) d \mathbf{y} .
$$

Since $\Omega_{r} \subset\left(B_{R_{0}} \backslash U^{*}\right)$ for all $r>R_{0}$ and $|\Delta \phi|$ is essentially bounded on $\Omega_{r}$, from equations (2.9), (2.17) and (2.18) it immediately follows that for $n \geq 3$,

$$
\left|u_{r}(\mathbf{x})-g_{n}(|\mathbf{x}|)\right|=\left|u_{r}(\mathbf{x})\right| \leq \frac{C_{0}}{|\mathbf{x}|^{n-2}} \quad \forall R_{0} \leq|\mathbf{x}|<r,
$$

where $C_{0}>0$ is a constant independent of $r$. For $n=2$, since $\Omega_{r} \subset B_{R_{0}}$, $\Delta\left[u_{r}(\mathbf{x})-g_{n}(|\mathbf{x}|)\right]=0$ on $B_{r} \backslash B_{R_{0}}$ by the first equation in (2.12). Again, note that $u_{r}(\mathbf{x})-g_{n}(|\mathbf{x}|)=0$ on $\partial B_{r}$ and $\left|u_{r}(\mathbf{x})-g_{n}(|\mathbf{x}|)\right| \leq \alpha\left(R_{0}\right)$ on $\partial B_{R_{0}}$. From the maximum principle applied to $u_{r}(\mathbf{x})-g_{n}(|\mathbf{x}|)$ restricted to $B_{r} \backslash B_{R_{0}}$, we conclude that equation (2.19) also holds for $n=2$ and the constant $C_{0}=\alpha\left(R_{0}\right)$. Therefore, by the triangle inequality and (2.19) we have

$$
\begin{array}{rlr}
\left|u_{\infty}(\mathbf{x})-g_{n}(|\mathbf{x}|)\right| & \leq\left|u_{\infty}(\mathbf{x})-u_{r_{j}}(\mathbf{x})\right|+\left|u_{r_{j}}(\mathbf{x})-g_{n}(|\mathbf{x}|)\right| \\
& \leq\left|u_{r_{j}}(\mathbf{x})-u_{\infty}(\mathbf{x})\right|+C_{0} /|\mathbf{x}|^{n-2} \quad \text { on } B_{R} .
\end{array}
$$

Fixing $R$ and sending $r_{j} \rightarrow \infty$ we get the third equation in (2.16) for all $n \geq 2$.

Finally we show the limiting minimizer $u_{\infty}$ must be unique. Assume that equations (2.15) and (2.16) are satisfied by a second function $u_{\infty}^{\prime} \in W_{l o c}^{2,2}\left(\mathbb{R}^{n}\right)$. Let $\Omega_{\infty}^{\prime}:=\left\{\mathbf{x} \in \mathbb{R}^{n}: u_{\infty}^{\prime}(\mathbf{x})=\phi(\mathbf{x})\right\}$ be the new coincident set. By the divergence theorem, we have for any $R>R_{0}$ and any $v \in K_{\infty}:=\left\{w \in W_{\text {loc }}^{1,2}\left(\mathbb{R}^{n}\right): w \geq \phi\right\}$,

$$
\begin{aligned}
& \int_{B_{R}} \nabla u_{\infty} \cdot \nabla\left(v-u_{\infty}\right) d \mathbf{x}-\int_{\partial B_{R}}\left(v-u_{\infty}\right) \mathbf{n} \cdot \nabla u_{\infty} d S \\
& =\int_{B_{R}}\left(-\Delta u_{\infty}\right)\left(v-u_{\infty}\right) d \mathbf{x} \\
& =\int_{\left\{\mathbf{x} \in B_{R}: u_{\infty}>\phi\right\}}\left(-\Delta u_{\infty}\right)\left(v-u_{\infty}\right) d \mathbf{x}+\int_{\left\{\mathbf{x} \in B_{R}: u_{\infty}=\phi\right\}}\left(-\Delta u_{\infty}\right)(v-\phi) d \mathbf{x} \geq 0,
\end{aligned}
$$

where $d S$ denotes the surface measure on $\partial B_{R}, \mathbf{n}$ is the outward normal of $d S$, and the inequality follows from (2.15). Clearly, equation (2.20) holds with $u_{\infty}$ replaced by $u_{\infty}^{\prime}$ as well:

$$
\int_{B_{R}} \nabla u_{\infty}^{\prime} \cdot \nabla\left(v-u_{\infty}^{\prime}\right) d \mathbf{x}-\int_{\partial B_{R}}\left(v-u_{\infty}^{\prime}\right) \mathbf{n} \cdot \nabla u_{\infty}^{\prime} d S \geq 0 \quad \forall v \in K_{\infty} .
$$

Since $u_{\infty}^{\prime}, u_{\infty} \in K_{\infty}$, adding equation (2.20) with $v=u_{\infty}^{\prime}$ to equation (2.21) with $v=u_{\infty}$, we obtain

$$
-\int_{B_{R}}\left|\nabla\left(u_{\infty}^{\prime}-u_{\infty}\right)\right|^{2} d \mathbf{x}+\int_{\partial B_{R}}\left(u_{\infty}-u_{\infty}^{\prime}\right) \mathbf{n} \cdot \nabla\left(u_{\infty}-u_{\infty}^{\prime}\right) d S \geq 0 .
$$

Further, by equations (2.16) and (2.1) we can express $u_{\infty}\left(u_{\infty}^{\prime}\right)$ as

$$
\begin{aligned}
u_{\infty}(\mathbf{x}) & =\int_{\mathbb{R}^{n}} \chi_{\Omega_{\infty}}(\mathbf{y}) \Delta \phi(\mathbf{y}) \Gamma(\mathbf{x}-\mathbf{y}) d \mathbf{y}+C_{1} \\
\left(u_{\infty}^{\prime}(\mathbf{x})\right. & \left.=\int_{\mathbb{R}^{n}} \chi_{\Omega_{\infty}^{\prime}}(\mathbf{y}) \Delta \phi(\mathbf{y}) \Gamma(\mathbf{x}-\mathbf{y}) d \mathbf{y}+C_{1}^{\prime}\right),
\end{aligned}
$$

Article submitted to Royal Society 
where $C_{1}, C_{1}^{\prime}$ are constants that are equal to zero if $n \geq 3$. Sending $R \rightarrow \infty$ in (2.22), by equations (2.23) and (2.1) we have $\lim _{R \rightarrow \infty} \int_{\partial B_{R}}\left(u_{\infty}-u_{\infty}^{\prime}\right) \mathbf{n} \cdot \nabla\left(u_{\infty}-\right.$ $\left.u_{\infty}^{\prime}\right) d S=0$ for $n \geq 3$. Thus, by (2.22) we obtain

$$
-\lim _{R \rightarrow \infty} \int_{B_{R}}\left|\nabla\left(u_{\infty}^{\prime}-u_{\infty}\right)\right|^{2} d \mathbf{x} \geq 0,
$$

which clearly implies that $u_{\infty}$ can be different from $u_{\infty}^{\prime}$ at most by a constant. From the last equation in (2.16) it follows that $u_{\infty}^{\prime}=u_{\infty}$ if $n \geq 3$. Below we show the same conclusion holds for $n=2$.

If $n=2$, by the last equation in (2.16), (2.23) and (2.1) we have

$$
\int_{\mathbb{R}^{n}} \chi_{\Omega_{\infty}}(\mathbf{y}) \Delta \phi(\mathbf{y}) d \mathbf{y}=\int_{\mathbb{R}^{n}} \chi_{\Omega_{\infty}^{\prime}}(\mathbf{y}) \Delta \phi(\mathbf{y}) d \mathbf{y}=-2 \pi Q .
$$

and

$$
\begin{aligned}
\left|u_{\infty}(\mathbf{x})-u_{\infty}^{\prime}(\mathbf{x})\right| \leq & \left|u_{\infty}(\mathbf{x})-g_{n}(|\mathbf{x}|)\right| \\
& +\left|u_{\infty}^{\prime}(\mathbf{x})-g_{n}(|\mathbf{x}|)\right| \leq 2 C_{0} \quad \forall|\mathbf{x}|>R_{0} .
\end{aligned}
$$

Therefore, again by equations (2.23) and (2.1) we have

$$
\left|\nabla\left[u_{\infty}(\mathbf{x})-u_{\infty}^{\prime}(\mathbf{x})\right]\right| \leq \frac{C}{|\mathbf{x}|^{2}} \quad \forall|\mathbf{x}|>R_{0},
$$

where $C>0$ is some constant independent of $\mathbf{x}$. From equations (2.26) and (2.27), it is clear that for $n=2$,

$$
\lim _{R \rightarrow \infty} \int_{\partial B_{R}}\left(u_{\infty}-u_{\infty}^{\prime}\right) \mathbf{n} \cdot \nabla\left(u_{\infty}-u_{\infty}^{\prime}\right) d S=0 .
$$

By sending $R \rightarrow \infty$ in equation (2.22), we again obtain equation (2.24), which implies that $u_{\infty}$ can be different from $u_{\infty}^{\prime}$ at most by a constant. If this constant is nonzero, one of the coincident sets $\Omega_{\infty}$ and $\Omega_{\infty}^{\prime}$ must be empty, which contradicts equation (2.25) since $Q>0$.

We remark that the uniqueness of the weak limit implies the convergence in equation (2.14) is in fact strong, see Rudin (1991). We summarize below.

Theorem 2.3. Consider the variational inequality problem (2.7) with an obstacle $\phi$ specified as above. Define the limiting minimizer $u_{\infty}$ and coincident set $\Omega_{\infty}$ as above. Then the interior of the coincident set $\Omega_{\infty} \subset B_{R_{0}}$ is an inclusion such that the overdetermined problem

$$
\left\{\begin{array}{lr}
\Delta u=\chi_{\Omega_{\infty}} \Delta \phi & \text { a.e. } \text { on } \mathbb{R}^{n} \\
\nabla \nabla u=\nabla \nabla \phi & \text { on } \Omega_{\infty} \backslash \partial \Omega_{\infty} \\
\left|u(\mathbf{x})-g_{n}(|\mathbf{x}|)\right| \leq \frac{C_{0}}{|\mathbf{x}|^{n-2}} & \text { for } \quad|\mathbf{x}| \geq R_{0}
\end{array}\right.
$$

is solved by $u=u_{\infty} \in W_{l o c}^{2, \infty}\left(\mathbb{R}^{n}\right)$, which also satisfies

$$
-\Delta u \geq 0, u \geq \phi, \quad \text { and } \quad-\Delta u(u-\phi)=0 \text { a.e. on } B_{R} \forall R>R_{0} \text {. }
$$

Further, if there is a second $u^{\prime} \in W_{l o c}^{2,2}\left(\mathbb{R}^{n}\right)$ that satisfies equation (2.29) and (2.28), then $u^{\prime}=u$. 
We remark that the last equation in (2.28) assures that a solution of (2.28) is the Newtonian potential (within an additive constant if $n=2$ ) induced by the source $\chi_{\Omega_{\infty}} \Delta \phi$.

\section{Solutions to the Eshelby conjectures}

In this section we present the details of the proofs of Theorems 1.2 and 1.3 and examples of special inclusions in various senses. Both the proofs and examples are derived from Theorem 2.3.

\section{(a) Proof of the Eshelby conjecture II (Theorem 1.2)}

To prove Theorem 1.2, by Lemma 2.1, equation (2.5), we see that it is sufficient to show that, if a connected inclusion $\Omega$ with Lipschitz boundary is such that the overdetermined problem

$$
\begin{cases}\Delta u=-\chi_{\Omega} & \text { on } \mathbb{R}^{n} \\ \nabla \nabla u=\mathbf{Q} & \text { on } \Omega \\ \left|u(\mathbf{x})-g_{n}(|\mathbf{x}|)\right| \leq \frac{C_{0}}{|\mathbf{x}|^{n-2}} & \text { on } \quad \mathbb{R}^{n} \backslash B_{R_{0}}\end{cases}
$$

admits a solution $u \in W_{\text {loc }}^{2,2}\left(\mathbb{R}^{n}\right)$ for some $\mathbf{Q} \in \mathbb{R}_{\text {sym }}^{n \times n}$ with $\operatorname{Tr}(\mathbf{Q})=-1$, then $\Omega$ must be an ellipsoid.

From equations (2.1) and (3.1) we have

$$
u(\mathbf{x})=-\int_{\mathbb{R}^{n}} \chi_{\Omega}(\mathbf{y}) \Gamma(\mathbf{x}-\mathbf{y}) d \mathbf{y}+C,
$$

where $C$ is a constant that vanishes if $n \geq 3$. By the divergence theorem, we have

$$
\int_{\mathbb{R}^{n}}(\nabla \nabla u)^{2} d \mathbf{x}=\int_{\mathbb{R}^{n}} \nabla \nabla u \Delta u d \mathbf{x}
$$

Since $\Omega$ is bounded and $\operatorname{Tr}(\mathbf{Q})=-1$, the left-hand side of (3.3) is always a positive definite matrix, which, by the first two equations in (3.1) and (3.3), implies the matrix $\mathbf{Q}$ is negative definite. Further, since $\Omega$ is a connected inclusion, there exists a quadratic function

$$
\phi(\mathbf{x})=\frac{1}{2}(\mathbf{x}-\mathbf{d}) \cdot \mathbf{Q}(\mathbf{x}-\mathbf{d})+h \text { such that } u(\mathbf{x})=\phi(\mathbf{x}) \quad \forall \mathbf{x} \in \Omega,
$$

where $\mathbf{d} \in \mathbb{R}^{n}$ and $h \in \mathbb{R}$.

We claim $u \geq \phi$ on $\mathbb{R}^{n}$. To show this, we notice that $\partial \Omega$ being Lipschitz continuous implies that for any unit vector $\mathbf{m} \in \mathbb{R}^{n}$,

$$
\mathbf{m} \cdot \nabla u(\mathbf{x})=-\int_{\mathbb{R}^{n}} \chi_{\Omega}(\mathbf{y}) \mathbf{m} \cdot \nabla \Gamma(\mathbf{x}-\mathbf{y}) d \mathbf{y}=\int_{\partial \Omega} \Gamma(\mathbf{x}-\mathbf{y}) \mathbf{m} \cdot \mathbf{n}(\mathbf{y}) d S(\mathbf{y}),
$$

where in the last equality, we have used the fact $\nabla_{\mathbf{x}} \Gamma(\mathbf{x}-\mathbf{y})=-\nabla_{\mathbf{y}} \Gamma(\mathbf{x}-\mathbf{y})$ and the divergence theorem. Note that above and subsequently, the gradient $\nabla$ is taken with respect to $\mathbf{x}$ unless it is stated otherwise. Thus, $\mathbf{m} \cdot \nabla u(\mathbf{x})$ is a single layer 
potential induced by a layer of charge with surface density $\mathbf{m} \cdot \mathbf{n}$ on $\partial \Omega$. By potential theory (see Kellogg 1929 page 160 for a classic treatment or Kenig 1994 page 54 and references therein for a modern viewpoint), it can be shown that

$$
\left.\nabla[\mathbf{m} \cdot \nabla u(\mathbf{x})]\right|_{\partial \Omega^{+}}-\left.\nabla[\mathbf{m} \cdot \nabla u(\mathbf{x})]\right|_{\partial \Omega^{-}}=[\mathbf{m} \cdot \mathbf{n}(\mathbf{x})] \mathbf{n}(\mathbf{x}) \quad \text { for a.e. } \mathbf{x} \in \partial \Omega,
$$

where $\partial \Omega^{+}\left(\partial \Omega^{-}\right)$means the limiting values approached from outside (inside) $\Omega$. Let $v_{\mathbf{m}}=\mathbf{m} \cdot[\nabla \nabla(u-\phi)] \mathbf{m}$. By the second equation in (3.1) and (3.4) we have

$\left.v_{\mathbf{m}}(\mathbf{x})\right|_{\partial \Omega^{+}}=\left.\mathbf{m} \cdot[\nabla \nabla u(\mathbf{x})-\mathbf{Q}] \mathbf{m}\right|_{\partial \Omega^{+}}=[\mathbf{m} \cdot \mathbf{n}(\mathbf{x})]^{2} \geq 0$ for a.e. $\mathbf{x} \in \partial \Omega$.

Also, from the representation formula (3.2) it is clear that

$$
v_{\mathbf{m}}(\mathbf{x}) \rightarrow-\mathbf{m} \cdot \mathbf{Q m}>0 \quad \text { as }|\mathbf{x}| \rightarrow \infty .
$$

Direct calculations reveal that $\Delta v_{\mathbf{m}}=0$ on $\mathbb{R}^{n} \backslash \bar{\Omega}$. By the maximum principle applied to $v_{\mathbf{m}}$ restricted to $\mathbb{R}^{n} \backslash \bar{\Omega}$ we conclude $v_{\mathbf{m}} \geq 0$ on $\mathbb{R}^{n} \backslash \bar{\Omega}$. Additionally, we note that for any $\mathbf{y} \in \mathbb{R}^{n} \backslash \bar{\Omega}$, there exist a point $\mathbf{x}_{0} \in \partial \Omega$ and a unit vector $\mathbf{m}$ such that $\mathbf{y}$ is an endpoint of the segment $\left\{\mathbf{x}_{0}+t \mathbf{m}: 0 \leq t \leq t_{0}\right\} \subset \mathbb{R}^{n} \backslash \bar{\Omega}$. Therefore, for $w(t)=u\left(\mathbf{x}_{0}+t \mathbf{m}\right)-\phi\left(\mathbf{x}_{0}+t \mathbf{m}\right)$ we have

$$
w(0)=0,\left.\quad \frac{d w(t)}{d t}\right|_{t=0}=0,\left.\quad \frac{d^{2} w(t)}{d t^{2}}\right|_{t=0}=v_{\mathbf{m}}(\mathbf{x}+t \mathbf{m}) \geq 0 \forall 0<t \leq t_{0},
$$

where we have used the fact $u(\mathbf{x}) \in C^{1}\left(\mathbb{R}^{n}\right)$, see Gilbarg and Trudinger (1983, page $54)$. By equation (3.6) we conclude $w\left(t_{0}\right)=u(\mathbf{y})-\phi(\mathbf{y}) \geq 0$. Thus, $u \in W_{l o c}^{2,2}\left(\mathbb{R}^{n}\right)$ satisfies the overdetermined problem (3.1) and equation (2.29) for the quadratic function $\phi$ which coincides with $u$ on $\Omega$.

Finally, from the explicit Newtonian potential $u_{E} \in W_{l o c}^{2, \infty}\left(\mathbb{R}^{n}\right)$ induced by the source $-\chi_{\Omega_{E}}$ on an ellipsoidal inclusion $\Omega_{E}$ (see the textbook of Kellogg 1929 for $n=3$ and a paper of Shahgholian 1991 for $n \geq 2$ ), it is known that for the quadratic function $\phi(\mathbf{x})=\frac{1}{2}(\mathbf{x}-\mathbf{d}) \cdot \mathbf{Q}(\mathbf{x}-\mathbf{d})+h$, there exists an ellipsoid, appropriately positioned in the space, such that the Newtonian potential $u_{E}$ induced by $-\chi_{\Omega_{E}}$ satisfies (within an additive constant if $n=2$ ) the overdetermined problem (3.1) and equation (2.29) for the same obstacle as in (3.4). By Theorem 2.3, we conclude that $u_{E}=u$ and $\Omega=\Omega_{E}$, which completes our proof of Theorem 1.2.

\section{(b) Proof of the Eshelby conjecture I in two dimensions (Theorem 1.3)}

In two dimensional space $(n=2)$, the Eshelby conjecture $\mathrm{I}$ is also true since it also implies the overdetermined problem (3.1) admits a solution. To see this, let us recall equations (1.2), (2.4) and (2.5). We will show equation (1.6) implies that the Newtonian potential $u$ of $-\chi_{\Omega}$ satisfies $\nabla \nabla u=$ const. on $\Omega$ if $n=2$, and so, Theorem 1.3 will follow by the same arguments as Theorem 1.2, see Section 3(a).

For any nonzero $\mathbf{P} \in \mathbb{R}^{2 \times 2}$, by choosing an appropriate coordinate system we can write it as $\mathbf{P}=\left[\begin{array}{cc}a & c \\ -c & b\end{array}\right]$, where $a^{2}+b^{2}+c^{2} \neq 0$. There are two possibilities that need separate attentions:

(i) If $a \neq b$, contracting $p$ and $i$ in (1.2), by equations (2.4) and (1.6), we have

$$
[\nabla \mathbf{v}(\mathbf{x}, \mathbf{P})]_{p p}=\frac{-1}{(2 \pi)^{n}\left(\mu_{1}+\mu_{2}+\lambda\right)} \int_{\mathbb{R}^{n}} \frac{a(\mathbf{k})_{1}^{2}+b(\mathbf{k})_{2}^{2}}{|\mathbf{k}|^{2}} g_{\Omega}(\mathbf{k}, \mathbf{x}) d \mathbf{k}
$$


is constant for all $\mathbf{x} \in \Omega$, where $g_{\Omega}(\mathbf{k}, \mathbf{x})=\int_{\Omega} \exp \left(i \mathbf{k} \cdot\left(\mathbf{x}-\mathbf{x}^{\prime}\right)\right) d \mathbf{x}^{\prime}$. By the inversion theorem, we also have

$$
\int_{\mathbb{R}^{n}} \frac{(\mathbf{k})_{1}^{2}+(\mathbf{k})_{2}^{2}}{|\mathbf{k}|^{2}} g_{\Omega}(\mathbf{k}, \mathbf{x}) d \mathbf{k}=\int_{\mathbb{R}^{n}} g_{\Omega}(\mathbf{k}, \mathbf{x}) d \mathbf{k}=(2 \pi)^{2} \chi_{\Omega}(\mathbf{x})
$$

Since $a \neq b,(\mathbf{k})_{i}^{2}$ for each $i=1,2$ can be written as a linear combination of $a(\mathbf{k})_{1}^{2}+b(\mathbf{k})_{2}^{2}$ and $(\mathbf{k})_{1}^{2}+(\mathbf{k})_{2}^{2}$. Thus,

$$
\int_{\mathbb{R}^{n}} \frac{(\mathbf{k})_{i}^{2}}{|\mathbf{k}|^{2}} g_{\Omega}(\mathbf{k}, \mathbf{x}) d \mathbf{k}=\text { const } \quad \text { on } \Omega \quad \forall i=1,2,
$$

which, by equation (2.3), implies the Newtonian potential $u$ induced by $-\chi_{\Omega}$ satisfies the Poisson equation (2.2) and

$$
\left\{\begin{array}{ll}
\frac{\partial^{2} u\left(x_{1}, x_{2}\right)}{\partial x_{1}^{2}}=A & \forall\left(x_{1}, x_{2}\right) \in \Omega \\
\frac{\partial^{2} u\left(x_{1}, x_{2}\right)}{\partial x_{2}^{2}}=B & \forall\left(x_{1}, x_{2}\right) \in \Omega
\end{array},\right.
$$

where $A, B \in \mathbb{R}$ are constants. Since $u\left(x_{1}, x_{2}\right)$ is an analytic function on the inclusion $\Omega$, by direct integration we see equation (3.10) implies all components of $\nabla \nabla u\left(x_{1}, x_{2}\right)$ are uniform on $\Omega$. Thus, Theorem 1.3 follows from Theorem 1.2 and Lemma 2.1 if $n=2$ and $a \neq b$.

(ii) If $a=b$, from equation (2.4), equations (1.2) and (1.6) imply

$\int_{\mathbb{R}^{n}}\left[\begin{array}{cc}a(\mathbf{k})_{1}^{2}+r c(\mathbf{k})_{1}(\mathbf{k})_{2} & a(\mathbf{k})_{1}(\mathbf{k})_{2}+r c(\mathbf{k})_{2}^{2} \\ a(\mathbf{k})_{1}(\mathbf{k})_{2}-r c(\mathbf{k})_{1}^{2} & a \mathbf{k}_{2}^{2}-r c(\mathbf{k})_{1}(\mathbf{k})_{2}\end{array}\right] \frac{g_{\Omega}(\mathbf{k}, \mathbf{x})}{|\mathbf{k}|^{2}} d \mathbf{k}=$ const. on $\Omega$

where $r=\frac{\mu_{1}+\mu_{2}+\lambda}{\mu_{1}}$. Combined with equation (3.8), one can write them as

$$
\int_{\mathbb{R}^{n}}\left[\begin{array}{ccc}
a & r c & 0 \\
-r c & a & 0 \\
0 & a & r c \\
0 & -r c & a \\
1 & 0 & 1
\end{array}\right]\left[\begin{array}{c}
(\mathbf{k})_{1}^{2} \\
(\mathbf{k})_{1}(\mathbf{k})_{2} \\
(\mathbf{k})_{2}^{2}
\end{array}\right] \frac{g_{\Omega}(\mathbf{k}, \mathbf{x})}{|\mathbf{k}|^{2}} d \mathbf{k}=\text { const. on } \Omega .
$$

Since $r \neq 0$ and $a^{2}+c^{2} \neq 0$, the rank of the $5 \times 3$ matrix inside the integral (3.11) is three, which again implies equation (3.10). This fact and the arguments for the previous case complete our proof for Theorem 1.3.

\section{(c) Existence of multiply-connected E-inclusions}

To prove Theorem 1.4, it is sufficient to show the existence of a multiplyconnected inclusion $\Omega$ such that the induced field $\nabla \mathbf{v}(\mathbf{x}, \mathbf{I})$ in (1.2) is uniform on $\Omega$. From Lemma 2.1, this is equivalent to the existence of a multiply-connected E-inclusion $\Omega$ such that the overdetermined problem (3.1) admits a solution in $W_{\text {loc }}^{2,2}\left(\mathbb{R}^{n}\right)$ for some $\mathbf{Q} \in \mathbb{R}_{\text {sym }}^{n \times n}$ with $\operatorname{Tr}(\mathbf{Q})=-1$. We claim there are many other non-ellipsoidal inclusions having this property. For reasons explained in Liu et al (2007), we call such special inclusions E-inclusions. We remark that E-inclusions 
include but are not limited to inclusions $\Omega$ such that $\nabla \nabla u$ in (2.3) are uniform on $\Omega$, see Liu et al (2007).

To construct such a multiply-connected E-inclusion, we consider piecewise quadratic obstacles

$$
\phi(\mathbf{x})=\sup \left\{\frac{1}{2}\left(\mathbf{x}-\mathbf{d}_{i}\right) \cdot \mathbf{Q}\left(\mathbf{x}-\mathbf{d}_{i}\right)+h_{i}: i=1, \cdots, N\right\}
$$

where $h_{1}, \cdots, h_{N} \in \mathbb{R}$ and $\mathbf{d}_{1}, \cdots \mathbf{d}_{N} \in \mathbb{R}^{n}$ are to be specified below. If the symmetric matrices $\mathbf{Q}$ is negative definite, it is easy to verify that the obstacle $\phi(\mathbf{x})$ defined in (3.12) satisfies all conditions required by Theorem 2.3, see Friedman (1982, page 44, Ex. 2). We then consider the variational inequality (2.7) with the obstacle (3.12). From the discussions in Section 2, a limiting minimizer $u_{\infty}$ is welldefined and we denote by $\Omega$ the interior of the coincident set $\left\{\mathbf{x} \in \mathbb{R}^{n}: u_{\infty}(\mathbf{x})=\right.$ $\phi(\mathbf{x})\}$. Theorem 2.3 implies that $\Omega$ is an E-inclusion such that the overdetermined problem (3.1) admits a solution in $W_{l o c}^{2, \infty}\left(\mathbb{R}^{n}\right)$. We now show that $\Omega$ can be multiplyconnected if the parameters $h_{1}, \cdots, h_{N} \in \mathbb{R}$ and $\mathbf{d}_{1}, \cdots \mathbf{d}_{N} \in \mathbb{R}^{n}$ in (3.12) are chosen appropriately. For instance, let $N=2, h_{1}=h_{2}=1$, and $\mathbf{d}_{1}=-\mathbf{d}_{2}$. From $\phi\left(\mathbf{d}_{1}\right)=\phi\left(\mathbf{d}_{2}\right)=1$ and the last equation in (2.28) we see $\Omega$ cannot be empty if $n \geq 3$. If $n=2$, the constant $Q>0$ in (2.9) and equation (2.25) assure $\Omega$ is non-empty. Note that $\nabla \nabla \phi$ is unbounded on the plane passing the origin and with normal $\mathbf{d}_{1}$. Thus, equation (2.10) implies $\Omega$ cannot intersect with this plane. From the symmetry of $\phi$, it can be seen that $\Omega$ has two components separated by this plane, and hence $\Omega$ is multiply-connected, see Fig. 1. We have thus completed the proof of Theorem 1.4 .

It is interesting to see what these E-inclusions look like and how much they resemble separate ellipsoids. So we consider the following numerical scheme to solve the variational inequality (2.7). If the constraint $u_{r} \geq \phi$ is neglected, the EulerLagrange equation of the variational problem (2.7) is the familiar boundary value problem:

$$
\Delta u_{r}=0 \quad \text { on } \quad B_{r} \quad \text { and } \quad u_{r}=g_{n}(r) \text { on } \partial B_{r} .
$$

According to the finite element method (see e.g. Kwon and Bang 2000), this boundary value problem can be discretized as

$$
\hat{K} \hat{u}=\hat{f} .
$$

where $\hat{u}$, a column vector, denotes the values of the potential $u_{r}$ at the nodal points in the finite element model, $\hat{K}$ and $\hat{f}$ are usually called the stiffness matrix and loads, respectively. Now let us take into account the discretized constraint $\hat{u} \geq \hat{\phi}$, where $\hat{\phi}$ are the values of the obstacle $\phi$ at the nodal points. Then the discrete version of the variational inequality (2.7) becomes the following quadratic programming problem:

$$
\min \left\{\hat{G}(\hat{u})=-\frac{1}{2} \hat{u} \cdot \hat{K} \hat{u}+\hat{f} \cdot \hat{u}: \hat{u} \geq \hat{\phi}\right\}
$$

which can be easily solved using standard solvers. The following computations use a mesh in a unit circle or sphere $(r=1)$ which is denser around the coincident set and has a total of around $10^{5}$ nodal points. The iterations are terminated when the relative difference between the values $\hat{G}(\hat{u})$ of two consecutive iterations is less 
than $10^{-10}$. With these parameters, the iterations converge within a few minutes on a personal computer. The resulting coincident set $\Omega_{r}$ includes all nodal points on which $|\hat{u}-\hat{\phi}|$ is less than $a \times 10^{-4}$, where $a$ is at the order of 1 . Since the convergence in (2.14) is in fact strong, presumably $\Omega_{r}$ would be a good approximation of the limiting coincident set $\left\{\mathbf{x}: u_{\infty}(\mathbf{x})=\phi(\mathbf{x})\right\}$ if the boundary of $\Omega_{r}$ is relatively far away from that of the unit ball $B_{1}$. Such properties of $\Omega_{r}$ can be realized by choosing small $h_{i}$ and $\left|\mathbf{d}_{i}\right|$ in (3.12) for the obstacle $\phi$.

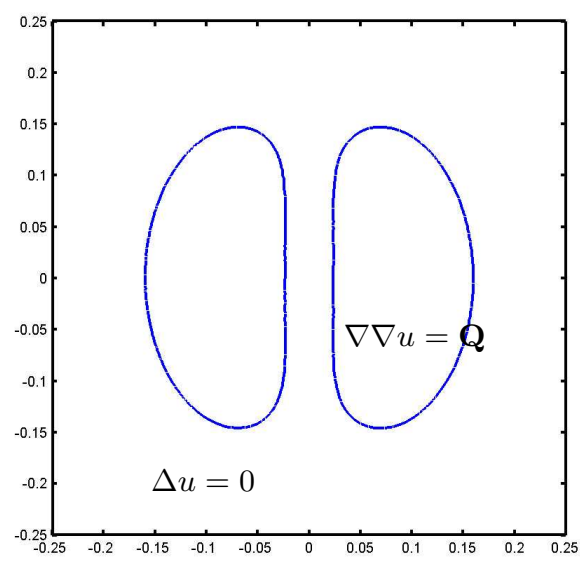

Figure 1. A two-component E-inclusion such that the overdetermined problem (3.1) admits a solution for $\mathbf{Q}=-\operatorname{diag}(1.5,1) / 2.5$.

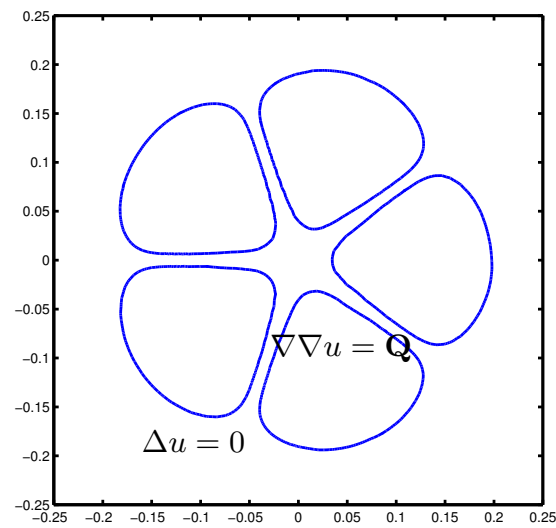

Figure 2. An E-inclusion with five-fold symmetry such that the overdetermined problem (3.1) admits a solution for $\mathbf{Q}=-\operatorname{diag}(1,1) / 2$.

If $N=1$ and $\mathbf{Q}$ is a negative definite matrix in (3.12), by Theorem 1.2 it is clear that the coincident set should be ellipses/ellipsoids in two/three dimensions. The numerical scheme is then verified by comparing the numerical results with the corresponding ellipses/ellipsoids in two/three dimensions. Below we show three examples of multiply-connected E-inclusions such that the overdetermined problem (3.1) admits a solution. The first two examples are calculated for the obstacle $\phi$ in (3.12) in two dimensions. Figure 1 shows a two-component E-inclusion such that the overdetermined problem (3.1) admits a solution for $\mathbf{Q}=-\operatorname{diag}(1.5,1) / 2.5$. The parameters in (3.12) are chosen to be $N=2, \mathbf{d}_{1}=[0,0.05], \mathbf{d}_{2}=-\mathbf{d}_{1}$, and $h_{1}=$ $h_{2}=0.02$. If the parameters in (3.12) are chosen to be $N=5, \mathbf{Q}=-\operatorname{diag}(1,1) / 2$, $\mathbf{d}_{i}=0.05 *[\cos (2 i \pi / 5), \sin (2 i \pi / 5)]$, and $h_{i}=0.025$ for $i=1, \cdots, 5$, we obtain an E-inclusion with five-fold symmetry, as shown in Fig. 2. This E-inclusion has five pedal-like components on which the second gradient of the induced potential is equal to $\mathbf{Q}=-\operatorname{diag}(1,1) / 2$, whereas the potential is harmonic outside.

The third example is a three dimensional E-inclusion such that the overdetermined problem (3.1) admits a solution for $\mathbf{Q}=-\operatorname{diag}(1,1,1) / 3$, see Fig. 3. Note that the mesh in this and following figures is not the actual mesh used in the computation but is merely used for visualization. Other parameters in (3.12) are $N=2$, $\mathbf{d}_{1}=[0,0,0.1], \mathbf{d}_{2}=-\mathbf{d}_{1}$, and $h_{1}=h_{2}=0.025$. The E-inclusion has two components which are symmetric about the plane $\left\{\mathbf{x}: x_{3}=0\right\}$. As the two dimensional 
example in Fig. 2, the boundaries of the two components become flatter as they come closer to each other. The front view of the lower component is plotted separately in Fig. 4, which shows a pedal-like area. According to symmetry, by rotating this area around the axis $\mathbf{e}_{3}=[0,0,1]$ we will obtain the lower component in Fig. 3 .

We remark that by changing the parameters in (3.12), we can construct a very large class of E-inclusions. The shapes, topology, the number of components, and the distances between various components of an E-inclusion can all be adjusted, see Liu et al (2007) for more examples in a periodic setting.

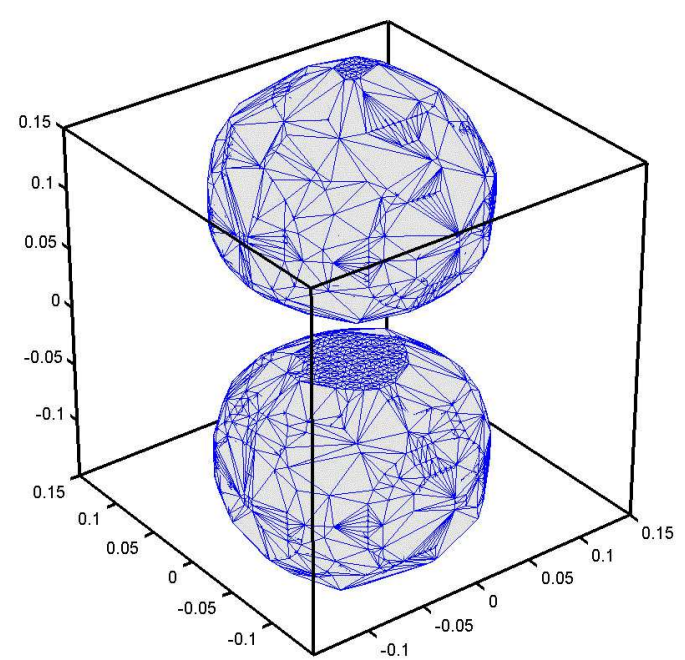

Figure 3. A two-component E-inclusion such that the overdetermined problem (3.1) admits a solution with $\mathbf{Q}=-\operatorname{diag}(1,1,1) / 3$, see Fig. 4 for front view.

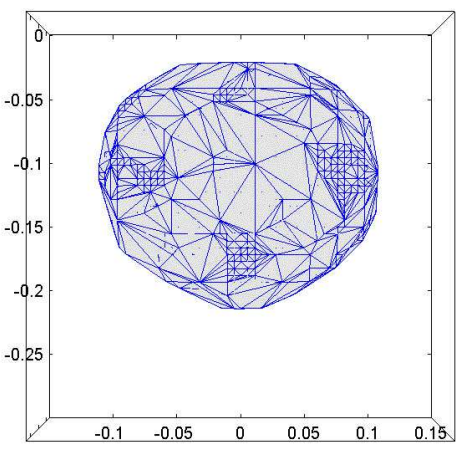

Figure 4. The front view of the lower component of Fig. 3.

\section{(d) Eshelby conjecture I for $n \geq 3$ (Theorem 1.5)}

We have shown that with $\mathbf{L}$ specified by (1.3) and (1.4), the Eshelby conjecture $\mathrm{I}$ is valid if $n=2$ or $n \geq 3$ and $\mathbf{P}=\mathbf{I}$, see Theorem 1.3, Theorem 1.2, and equations (2.6) and (2.5). However, if $n \geq 3$, the Eshelby conjecture I may not be valid depending on the tensor $\mathbf{L}$ and matrix $\mathbf{P}$. Below we construct a non-ellipsoidal inclusion $\Omega \subset \mathbb{R}^{3}$ with smooth boundary having the property that

$$
\nabla \mathbf{v}(\mathbf{x}, \mathbf{P})=\text { const. } \quad \text { on } \Omega \text { for } \mathbf{P}=\operatorname{diag}(1,0,0),
$$

where $\nabla \mathbf{v}(\mathbf{x}, \mathbf{P})$ is given by (1.2) and $\mathbf{L}$ is specified by (1.3) and (1.4) with $\mu_{2}+\lambda=0$. By equations (2.4) and (2.3), this is equivalent to the existence of a non-ellipsoidal inclusion $\Omega$ with smooth boundary such that the Newtonian potential $u$ induced by $-\chi_{\Omega}$ satisfying

$$
\frac{\partial^{2} u(\mathbf{x})}{\partial x_{1} x_{i}}=\text { const. } \quad \text { on } \Omega \quad \forall i=1,2,3 .
$$


We use Theorem 2.3 to construct such a domain in $\mathbb{R}^{3}$. We need to carefully define our obstacle such that the second equation in (2.28) implies (3.15) without the first one being violated. Let $\hat{\phi}: \mathbb{R}^{2} \rightarrow \mathbb{R}$ be

$$
\hat{\phi}\left(x_{2}, x_{3}\right)= \begin{cases}Q \log \left(x_{2}^{2}+x_{3}^{2}\right)^{1 / 2} & \text { if }\left(x_{2}^{2}+x_{3}^{2}\right)^{1 / 2}>1 \\ 0 & \text { if }\left(x_{2}^{2}+x_{3}^{2}\right)^{1 / 2} \leq 1\end{cases}
$$

and $\phi: \mathbb{R}^{3} \rightarrow \mathbb{R}$ be

$$
\phi(\mathbf{x})=-\frac{1}{6}\left[x_{1}^{2}+\left(x_{2}-a\right)^{2}+\left(x_{3}-a\right)^{2}\right]+\hat{\phi}\left(x_{2}-b, x_{3}-b\right)+h,
$$

where the constants $Q>0$ and $a, b, h \in \mathbb{R}$ are to be determined. Direct calculations reveal that if $a=-1, b=-3 Q / 2, h$ and $Q$ are appropriately chosen, say, $h=-2.54$ and $Q=2$, the obstacle $\phi$ defined in (3.16) enjoys the following properties:

(i) $\phi$ satisfies all properties listed on page 5;

(ii) $\phi<0$ on $U_{2}:=\left\{\mathbf{x} \in \mathbb{R}^{n}:\left(x_{2}+3\right)^{2}+\left(x_{3}+3\right)^{2} \leq 1\right\}$;

(iii) On $U_{1}:=\left\{\mathbf{x} \in \mathbb{R}^{n}:\left(x_{2}+3\right)^{2}+\left(x_{3}+3\right)^{2}>1\right\}$, we have $\Delta \phi=-1$ and

$$
\begin{aligned}
\nabla \nabla \phi(\mathbf{x})=-\mathbf{I} / 3 & +\frac{1}{\rho^{4}}\left[\left(\rho^{2}-2\left(x_{2}+3\right)^{2}\right) \mathbf{e}_{2} \otimes \mathbf{e}_{2}+\left(\rho^{2}-2\left(x_{3}+3\right)^{2}\right) \mathbf{e}_{3} \otimes \mathbf{e}_{3}\right. \\
& \left.+2\left(x_{2}+3\right)\left(x_{3}+3\right)\left(\mathbf{e}_{2} \otimes \mathbf{e}_{3}+\mathbf{e}_{3} \otimes \mathbf{e}_{2}\right)\right]
\end{aligned}
$$

where $\rho=\sqrt{\left(x_{2}+3\right)^{2}+\left(x_{3}+3\right)^{2}}$, and $\mathbf{e}_{1}, \mathbf{e}_{2}, \mathbf{e}_{3}$ denote the unit vectors which we use to define our rectangular coordinates $\mathbf{x}=\left(x_{1}, x_{2}, x_{3}\right)$.

(iv) $\phi(0)=-2.54-1 / 3+2 \log (3 \sqrt{2}) \approx 0.017>0$, and on $U_{1}$,

$\nabla \phi(\mathbf{x})=0$ if and only if $\mathbf{x}=0 \quad$ and $\left.\quad \nabla \nabla \phi(\mathbf{x})\right|_{\mathbf{x}=0}$ is negative definite.

Therefore, the maximum of $\phi(\mathbf{x})$ is attained only at 0 .

We now apply Theorem 2.3 with an obstacle defined in (3.16) satisfying all conditions listed above. Immediately, we obtain the existence of a non-ellipsoidal inclusion $\Omega$ such that the Newtonian potential $u$ induced by $-\chi_{\Omega}$ satisfying

$$
\nabla \nabla u=\nabla \nabla \phi \quad \text { on } \Omega .
$$

That is, the overdetermined problem (2.28) admits a solution $u \in W_{l o c}^{2, \infty}\left(\mathbb{R}^{3}\right)$ for the obstacle $\phi$ in (3.16). Further, since $\phi$ is smooth restricted to $\{\mathbf{x}: \phi(\mathbf{x})>0\}$, it can be shown that the coincident set has smooth boundary, see Friedman (1982, Ch. 2). From equations (3.17) and (3.18), we see $\partial^{2} u(\mathbf{x}) / \partial x_{1} x_{i}$ satisfy equation (3.15) for $i=1,2,3$. This completes our proof of Theorem 1.5.

The numerical scheme described in Section 3(c) can be used to calculate these inclusions $\Omega$. Figure 5 shows such an example, which is calculated with the obstacle (3.16). The parameters in (3.16) are chosen to be

$$
h=-2.54, \quad a=-1, \quad b=-3 \quad \text { and } \quad Q=2 .
$$




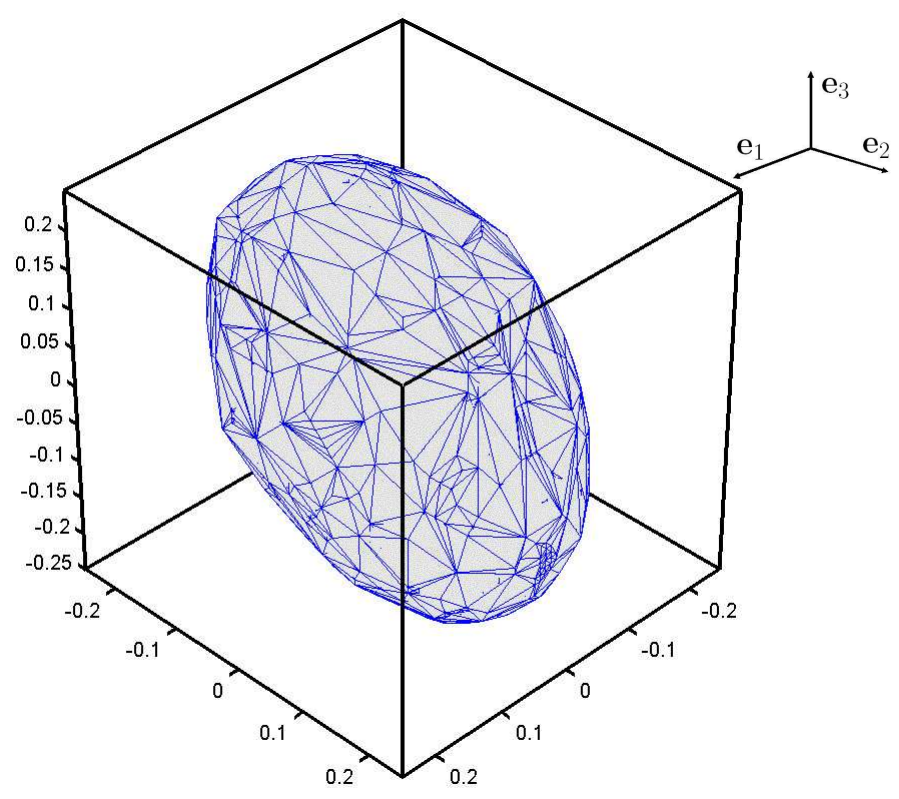

Figure 5. An inclusion whose Newtonian potential satisfies equations (3.18) and (3.17), and hence (3.15). The three orthographic views are shown in Fig. 6-Fig. 8.

The inclusion in Fig. 5 might appear like an ellipsoid, but in fact it cannot be an ellipsoid since not all components of $\nabla \nabla u$ are uniform on the inclusion, see equations (3.18) and (3.17). The three orthographic views are shown in Fig. 6Fig. 8. The view in Fig. 6 is from the direction $\mathbf{e}_{1}$ or from the left-hand side of Fig. 5 shows approximately an ellipse. The views in Fig. 7 and 8 are from the directions of $\mathbf{e}_{2}$ and $\mathbf{e}_{3}$ or the right-hand side and top of Fig. 5 show approximately circular areas. We are not aware of any kind of familiar geometry that can give rise to three orthographic views as in Fig. 6-Fig. 8. It is more or less like the shape one would obtain by squashing a ball non-uniformly in $\mathbf{e}_{2}+\mathbf{e}_{3}$ direction.

\section{Summary and discussions}

We have presented the solutions of the Eshelby conjectures interpreted in two different senses. The method in the paper relies on two key observations: (i) for tensors of form specified by (1.3), the vectorial equation (1.1) is solved by the gradient of the Newtonian potential, see Lemma 2.1, and (ii) solving the variational inequality (2.7) can produce special inclusions such that certain overdetermined problem admits a solution. From the established theory about variational inequalities, the Eshelby conjecture II, restricted to connected Lipschitz inclusions, follows from the uniqueness of the solution of the variational inequality (2.7) for a given quadratic obstacle, whereas by choosing other kinds of obstacles we are able to construct various special inclusions for which the desired overdetermined problems admit solutions. A nu- 


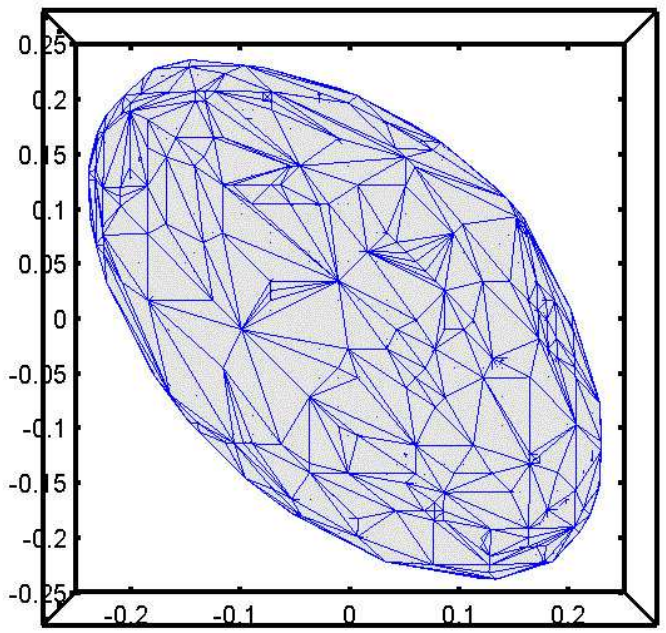

Figure 6. View of Fig. 5 from $\mathbf{e}_{1}$ direction.

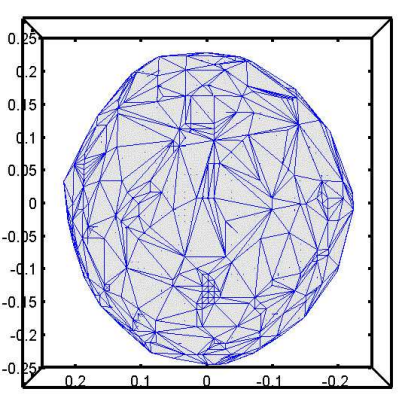

Figure 7. View of Fig. 5 from $\mathbf{e}_{2}$ direction.

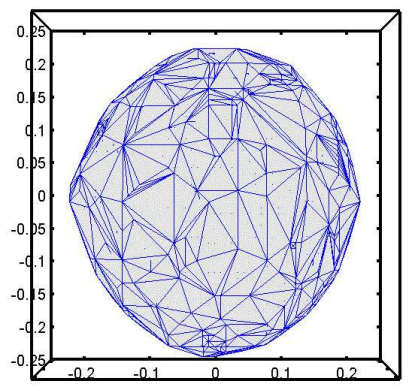

Figure 8. View of Fig. 5 from $\mathbf{e}_{3}$ direction.

merical scheme has been implemented to calculate these special inclusions discussed above, see Fig. 1-Fig. 8.

Note that in view of equation (1.1), the preceding arguments can be extended to tensors

$$
\left(\mathbf{L}^{\prime}\right)_{p i q j}=(\mathbf{G})_{r p}(\mathbf{G})_{s q}(\Lambda)_{i k}(\Lambda)_{j l}(\mathbf{L})_{r k s l}
$$

by a linear transformation

$$
\mathbf{x} \longrightarrow \mathbf{x}^{\prime}=\Lambda^{-1} \mathbf{x} \quad \text { and } \quad \mathbf{v} \longrightarrow \mathbf{v}^{\prime}=\mathbf{G}^{-1} \mathbf{v}
$$

where $\mathbf{G}, \Lambda \in \mathbb{R}^{n \times n}$ are invertible and $\mathbf{L}$ is of form (1.3). Further, through a refined calculation on the inverse of the matrix $(\mathbf{L})_{p i q j}(\mathbf{k})_{i}(\mathbf{k})_{j}$, we can extend the results to tensors that satisfy $(m=n)$

$$
(\mathbf{L})_{p i q j}(\mathbf{k})_{i}(\mathbf{k})_{j}(\mathbf{k})_{q}=\kappa|\mathbf{k}|^{2}(\mathbf{k})_{p} \quad \forall \mathbf{k} \in \mathbb{R}^{n}
$$

for some $\kappa>0$, see details in Liu et al (2007). The linear transformation (4.1) can be again applied to general $\mathbf{L}$ of form (4.2) and further extend the applicability of the preceding arguments. The reader is invited to formulate the precise statements corresponding to Theorem 1.2-Theorem 1.5 for tensors $\mathbf{L}$ of these forms.

Finally, a few remarks are in order regarding other applications of the variational inequality (2.7). First of all, in view of the applications of the Eshelby's solution (Eshelby 1957) for an ellipsoid in the theories of micromechanics, composites and fracture mechanics, by Theorem 1.4 we immediately extend these applications to multiply-connected E-inclusions as shown in Fig. 2 and Fig. 3 if the eigenstress is dilatational and the matrix phase is isotropic. For instance, we can show that a 
solution of the homogeneous Eshelby inclusion problem (1.1) also solves the corresponding inhomogeneous Eshelby inclusion problem, see Eshelby (1957) and Liu et al (2007). By a similar argument as in Roitburd (1986), if the interfacial energy is neglected, we can show that these multiply-connected E-inclusions, together with ellipsoids, are equilibrium shapes of inhomogeneous precipitates in alloys under some hypotheses on the mismatch strain and material properties. Moreover, if the problem of reducing the stress concentration around a hole in an elastic body is considered, depending on the external loading, boundary conditions and material properties, the variational inequality (2.7) with appropriate obstacles can be used to determine the optimal shapes of the holes with least stress concentration factors, see Lipton (2005). A closely related property of these optimal shapes in the context of composites, as shown in Liu et al (2007), is that they attain the optimal Hashin-Shtrikman bounds. In conclusion, as illustrated by the solutions to the Eshelby conjectures, the consideration of the variational inequality (2.7) can be useful in solving many physical problems and in particular those problems in which the shapes of the inclusions play an important role.

The author gratefully acknowledges the financial support of the US Office of Naval Research through the MURI grant N00014-06-1-0730.

\section{References}

Asaro, R. J. \& Barnett, D. M., 1975. The non-uniform transformation strain problem for an anisotropic ellipsoidal inclusion. J. Mech. Phys. Solids 23:77-83.

Bhattacharya, K. \& Li, J., 2001. Domain patterns, texture and macroscopic electromechanical behaviors of ferroelectrics. Workshop on Fundamental Physics of Ferroelectrics.

Brown, W. F., 1962. Magnetostatic principles in ferromagnetism. Amsterdam: NorthHolland Publishing Company.

Cherepanov, G. P., 1974. Inverse problems of the plate theory of elasticity. J. Appl. Math. Mech., PMM 38:963-979.

Christensen, R., 1979. Mechanics of Composite Materials. New York: Academic Press.

Dive, P., 1931. Attraction des ellipsoides homogènes et réciproques d'un théorème de Newton. Bull. Soc. Math. France 59:128-140.

Eshelby, J. D., 1957. The determination of the elastic field of an ellipsoidal inclusion and related problems. Proc. R. Soc. London, Ser. A 241:376-396.

Eshelby, J. D., 1961. Elastic inclusions and inhomogeneities. I.N. Sneddon and R. Hill (Eds.). Progress in Solid Mechanics II, North Holland: Amsterdam, 89-140.

Friedman, A., 1982. Variational principles and free boundary problems. New York : Wiley.

Gilbarg, D. \& Trudinger, N. S., 1983. Elliptic partial differential equations of second order. New York: Springer-Verlag.

Kang, H. \& Milton, G. W., 2007. Solutions to the conjectures of Pólya-Szegö and Eshelby. Arch. Ration. Mech. Anal. In press.

Kang, H., Kim, E., \& Milton, G. W., 2007. Inclusion pairs satisfying Eshelby's uniformity property. Submitted to SIAM J. Appl. Math.

Kellogg, O. D., 1929. Foundations of potential theory. New York : Dover Publications, INC.

Kenig, C. E., 1994. Harmonic analysis techniques for second order elliptic boundary value problems. AMS: Providence.

Khachaturyan, A. G., 1983. Theory of structural transformations in solids. New York: Wiley. 
Kinderlehrer, D. \& Stampacchia, G., 1980. An introduction to variational inequalities and their applications. New York: Academic Press.

Kwon, Y. W. \& Bang, H., 2000. The finite element method using MATLAB. Boca Raton, Fla. : CRC Press.

Lee, J. K. \& Johnson, W. C., 1977. Elastic strain energy and interactions of thin square plates which have undergone a simple shear. Scr. Metall. 11:477-484.

Lipton, R., 2005. Optimal lower bounds on the hydrostatic stress amplification inside random two-phase elastic composite. J. Mech. Phys. solids 53:2471-2481.

Liu, L. P., James, R. D. \& Leo, P. H., 2006. Magnetostrictive composites in the dilute limit. J. Mech. Phys. Solids 54:951-974.

Liu, L. P., James, R. D. \& Leo, P. H., 2007. New extremal inclusions and their applications to two-phase composites. Submitted to Archive for Rational Mechanics Analysis.

Markenscoff, X., 1998a. Inclusions with constant eigenstress. J. Mech. Phys. Solids 46:2297-2301.

Markenscoff, X., 1998b. On the shape of the Eshelby inclusions. J. elasticity 44:163-166.

Maxwell, J. C., 1873. A treatise on electricity and magnetism. Oxford, United Kindgdom: Clarendon Press.

Milton, G. W., 2002. The Theory of Composites. Cambridge University Press.

Mura, T., 1987. Micromechanics of Defects in Solids. Martinus Nijhoff.

Mura, T., 2000. Some new problems in the micromechanics. Materials Science and Engineering A 285:224-228.

Mura, T., Shodja, H. M., Lin, T. Y., Safadi, A. \& Makkawy, A., 1994. The determination of the elastic field of a pentagonal star shaped inclusion. Bull. tech. Univ. Istanbul 47:267-280.

Nikliborc, W., 1932. Eine bemerkung über die Volumpotentiale. Math. Zeit. 35:625-631.

Poisson, S. D., 1826. Second mémoire sur la théorie de magnetismé. Mémoires de l'Académie royale des Sciences de l'Institut de France 5:488-533.

Pólya, G. \& Szegö, G., 1951. Isoperimetric Inequalities for polarization and virtual mass, Annals of Mathematical Studies, Number 27. Princeton University Press.

Rodin, G., 1996. Eshelby's inclusion problem for polygons and polyhedra. J. Mech. Phys. Solids. 44:1977-1995.

Roitburd, A., 1986. Phase equilibrium in solids. Soviet Phys Solid State 28:1716-1718.

Ru, C. Q. \& Schiavone, P., 1996. On the elliptic inclusion in anti-plane shear. Math. Mech. Solids 1:327-333.

Rudin, W., 1991. Functional anlysis. New York: McGraw-Hill.

Sendeckyj, G. P. ,1970. Elastic inclusion problems in plane elastostatics. Int. J. Solids Structures 6:1535-1543.

Shahgholian, H., 1991. On the Newtonian potential of a heterogeneous ellipsoid. SIAM Journal on Mathematical Analysis 22:1246-1255.

Wu, L. \& Du, S. Y., 1995. The elastic field caused by a circular cylindrical inclusion-Part I. J. Appl. Mech 62:579-584. 